\title{
Preface: Special Session SpS17 Light Pollution: Protecting Astronomical Sites and Increasing Global Awareness Through Education
}

The issue of Light Pollution was a major concern of the International Astronomical Union; during the IAU General Assembly in the city of Rio de Janeiro in 2009. A resolution was unanimously adopted (Resolution B5) to support the need to preserve the night sky and the right to see stars. With the increasing use of artificial light at night posing a growing threat to the visibility of the night sky, this Special Session at the XXVIIIth General Assembly highlighted technical aspects of astronomical site protection and the educational aspects of increasing global awareness on issues concerning light pollution.

The primary responsibility for the disappearance of stars is peoples lack of knowledge. Recognizing the problem, studying its causes, and assessing options is part of the solution. Several topics for the session examined the impact of various approaches of education and public outreach on issues concerning light pollution and its impact on our world. To accomplish this, we present invited presentations by ecologists, amateur and professional astronomers, professional educators at schools, universities, planetaria, science centers and nature centers, as well as people in the media, landscape astrophotography, the medical field and lighting design. Contributions on how to improve our handling of language and cultural barriers, review progress being made by outreach and citizen-science programs such as GLOBE at Night, Global Astronomy Months Dark Skies Awareness, One Star at a Time, the Starlight Initiative and the educational resources offered through the International Dark-Sky Association are also present in these pages.

Protecting sites (including observatories) and slowing the encroachment of light pollution requires engaging the public on several levels. These actions include producing long-term sky brightness data intercomparable with broader public monitoring programs; taking opportunities to educate the public about the value of dark sky preservation; interacting with policy makers and public agencies ranging from localities to the UN to provide legal protection and enforcement for dark sky zones; and interacting with lighting engineers to define dark-sky preserving products and to encourage their deployment. Rapidly advancing technology and associated promotion is leading to deployment of blue-rich artificial light sources that threaten to impact a spectral region previously left relatively untouched. Exploring the nature, possible impact, and potential mitigation of this trend has been a timely aspect of this session.

The knowledge of starry sky represented for our ancestors the basis for survival on Earth, contributed to the development of science in all areas and was a source of inspiration for humanity. This heritage is being lost; to recover it for us and future generations is everyone's responsibility, because we know that individuals approach to the Cosmos today is still of the same importance and magnitude as in antiquity.

Richard Green, and Beatriz García, Constance Walker and Xue Sui Jian, co-chairs SOC, Rosa M. Ros, Elizabeth Álvarez, Magda Stavischi and Scott Kardel, SOC-Editors

December 31, 2012 